\title{
Preliminary Sizing Study of Ares-I and Ares-V Liquid Hydrogen Tanks
}

Stanley T. Oliver ${ }^{1}$

NASA Marshall Space Flight Center, Huntsville, AL

David W. Harper ${ }^{2}$

Boeing Huntsville Design Center, Huntsville, AL

Extended Abstract of Proposed Paper for the 53 ${ }^{\text {rd }}$ AIAA/ASME/ASCE/AHS/ASC Structures, Structural Dynamics, and Materials Conference, April 23-26, 2012, Honolulu, Hawaii

Category: Special Session - Developing the Next Generation of Shell Buckling Design Factors and Technologies. POC Dr. Mark W. Hilburger

\section{INTRODUCTION}

A FORTRAN optimization program was used to size the Ares-I liquid hydrogen (LH2) tank for minimum weight considering a less conservative shell buckling knockdown factor (SBKF) and permutations of specific design requirements. The purpose was to illustrate the potential impacts on tank weight due to the design requirements and less conservative SBKF.

\section{BACKGROUND}

NASA SP-8007 [1] provides recommended practices to determine the critical buckling load of circular cylinders. The closed-form analysis methodology and SBKF approach represented a lower-bound of test data. This lower-bound was established with a reduced understanding of cylinder construction or testing methodologies. With significant improvements in computers and nonlinear analysis tools, more accurate buckling predictions are possible by accounting for construction sensitivities [2] and testing methodology. This improved accuracy can therefore reduce the SBKF applied to the critical buckling load of circular cylinders.

The design requirements were established to mitigate safety concerns and add robustness to the design. The three design requirements considered in this study were:

1. Fail-safe loss of ullage pressure (emergency condition),

2. Fixed skin thickness to carry pressure load,

3. Acceptance proof test.

The sudden loss of ullage pressure during first stage ascent was considered a fail-safe emergency condition. Designing for the sudden loss of pressure ensured the tank would not collapse and

\footnotetext{
${ }^{1}$ Aerospace Engineer.

${ }^{2}$ Structural Analyst.
} 
allow for the initiation of the launch abort system. Fail-safe emergency conditions were subject to an ultimate factor of safety of 1.0 on design loads [3].

Robustness was added to the design by requiring the tank's skin to carry the entire pressure loads. This was to ensure that the future developed fracture properties and supporting fracture analyses would not yield fracture concerns associated with the tank's skin.

The third requirement was the acceptance proof test. Pneumatic and possibly mechanical loads were required to stress the tank above the maximum limit principal stress experienced in flight.

\section{DESIGN CRITERIA}

The geometry of the integrally machined barrel panels was limited to an orthogrid configuration with stringers and ribs oriented longitudinally and circumferentially respectively. The tank's skin was the pockets between the stringers and ribs. The overall plate thickness was limited to typical raw-plate stock for Aluminum-Lithium alloy 2195. A manufacturing constraint associated with bump-forming the orthogrid panels required that the ratio of the height of a rib or stringer to its thickness be equal to or less than $10(\mathrm{~h} / \mathrm{t}<=10)$. An arbitrary constraint limited the circumferential rib spacing to 20 inches. This upper-bound was established to allow the influence and trends of the other geometry variables to be more recognizable in the results.

For the compressive loading conditions, pressure stabilization was allowed using the minimum pressure and a factor of safety of 1.0. Local and global buckling was not allowed at ultimate load. For the tensile cases, maximum pressure was combined with the tensile mechanical loads and appropriate factors of safety.

The tensile and compressive failure modes evaluated were:

1. Pocket or skin principal stresses (yield and ultimate)

2. Rib principal stresses (yield and ultimate)

3. Stringer principal stresses (yield and ultimate)

4. Pocket or skin buckling

5. Rib buckling

6. Stringer buckling

7. Overall cylinder buckling

The vehicle flight loads were derived from the vehicle axial, shear and bending moments, combined to yield the design limit compressive and tensile mechanical line loads. The maximum and minimum pressures were considered, but the head pressure associated with the liquid hydrogen was ignored for this study due to its small contribution. 


\section{APPROACH}

The permutations of the three design requirements results in eight possible design studies:

1. Fail-safe loss of pressure, fixed skin thickness, and with a proof test

2. Fail-safe loss of pressure, fixed skin thickness, and no proof test

3. Fail-safe loss of pressure, optimized skin thickness, and with a proof test

4. Fail-safe loss of pressure, optimized skin thickness, and no proof test

5. Flight only, fixed skin thickness, and with a proof test

6. Flight only, fixed skin thickness, and no proof test

7. Flight only, optimized skin thickness, and with a proof test

8. Flight only, optimized skin thickness, and no proof test

The first four studies investigate the fail-safe loss of pressure condition; the flight loads are not considered. The last four studies consider the flight loads, but not the fail-safe condition. The fixed skin thickness option enforces a constant (minimum) skin thickness based on hoop stress, and the optimized skin thickness option allows the skin thickness to be determined by the optimizer program. The proof test was an additional load case and only impacted the tensile failure modes.

The approach to the study used SP-8007 to establish a baseline design for each of the eight cases, and then continued re-optimizing each case using a progressively improved shell buckling knockdown factor.

\section{Optimizing Program}

A FORTRAN 77 computer program [4] was utilized to obtain the minimum weight designs subjected to the constraints previously described. The orthogrid design parameters were skin thickness, stringer spacing, rib spacing, stringer width, rib width, and stiffener height. The program could process load cases comprised of vehicle mechanical loads, ullage pressures, head pressures, temperatures, load case specific factors of safety, and temperature-dependent material properties. It operates on the orthogid design parameters, incrementing through a user-defined vector space of parameter values, calculating margins of safety, and sorting through the results to find an orthogrid configuration that maintained positive margins yet had the lowest weight.

\section{RESULTS}

The results contained herein represent the barrel sections of the Ares-I LH2 tank. Domes, weld lands, rings, etc. are not considered for this study. Therefore, the meaning of tank weight in this section pertains only to the barrel panels.

The normalized Ares-I LH2 tank weight as a function of stability knockdown factor is shown in Figure 1. It should be noted that there are six results curves shown in Figure 1, but there were 
eight possible design studies. For the cases with a fixed skin thickness, the proof test has no impact on the weights. Therefore, these cases yield the same results, and the legend in the graph indentifies the two corresponding cases as combined for both the fail-safe and flight design cases. In addition, the baseline SBKF values for barrels were typically lower than 0.65 , but 0.65 represents a common SBKF for all barrels for all studies.

There are two distinct groupings in Figure 1, the closely spaced fail-safe grouping and the more widely spaced flight grouping. The fail-safe grouping's weight is higher because the optimization could not utilize pressure stabilization; whereas for flight, minimum pressure stabilization was sufficient to provide substantial benefit, even with the higher ultimate factors of safety. Enforcing a constant skin thickness did result in a heavier tank compared to allowing the thickness to be determined through optimization. This was especially true for the flight studies. The results show that the proof test does result in a slightly heavier tank (8\%) for the flight cases. For the fail-safe cases with optimization allowed for skin thickness, the proof test begins to be a design driver for SBKF values greater than 0.75 .

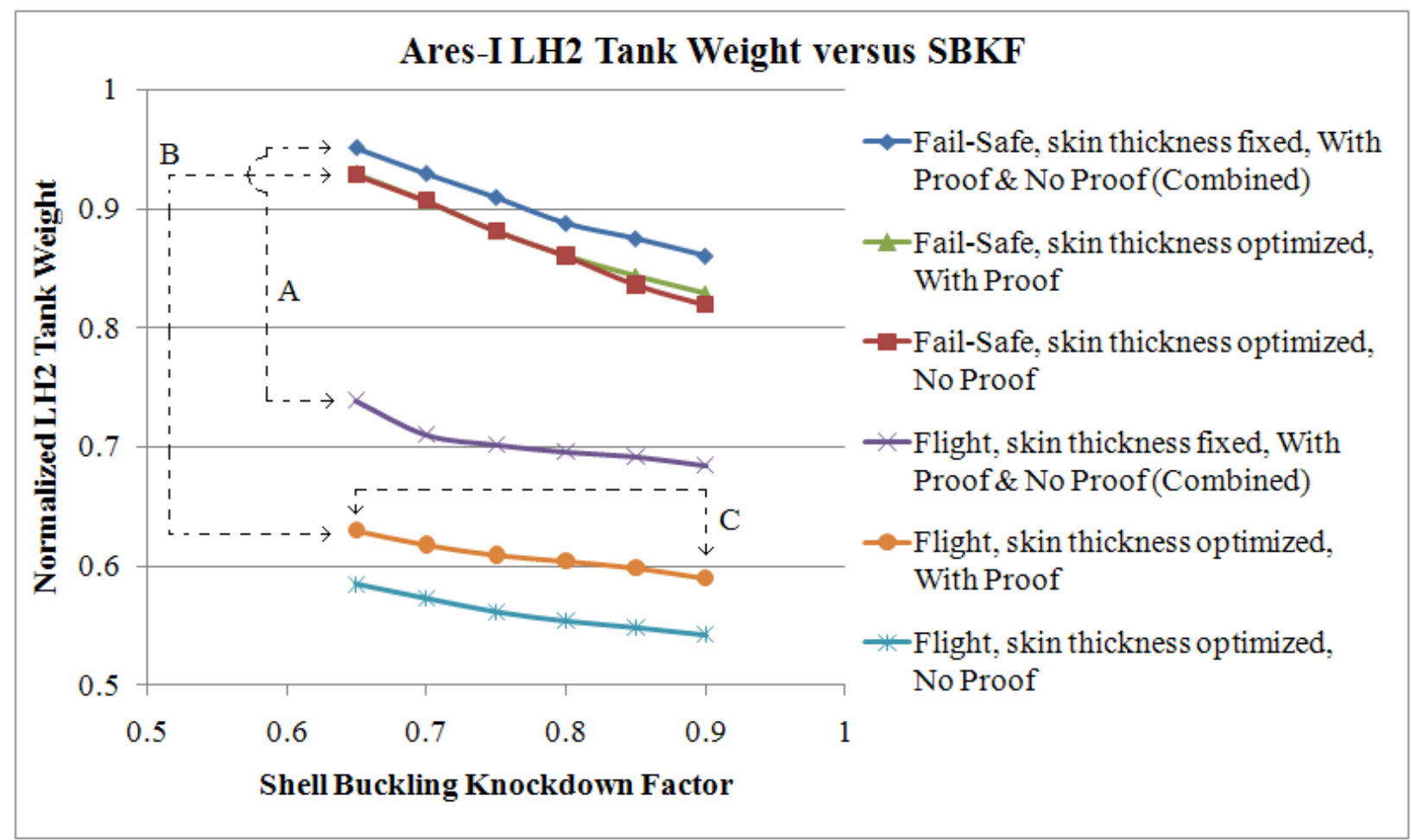

Figure 1 Optimization results for LH2 tank for various design considerations.

Surveying the Ares-I LH2 tank normalized results for a given stability knockdown factor, 0.65 for example, shows that the fail-safe requirement for the fixed skin thickness designs could result in a $28 \%$ increase in weight over the flight design for the fixed skin thickness (comparison A). The same comparison can be made for maintaining a proof test and allowing the skin thickness 
to vary; implementing the fail-safe requirement results in a weight increase of $47 \%$ over the corresponding flight design (comparison B).

Another study looked at the effect of utilizing a less conservative SBKF. For a flight case, allowing the skin thickness to vary and accounting for a proof test, the potential weight savings of designing for a SBKF of 0.90 compared to 0.65 was $6 \%$ (comparison C). One can see from the graph that the effect of SBKF is less for the flight cases than the fail-safe cases (observed slope of data). One potential reason is pressure stabilization during flight has not only the benefit of reduced axial load, but also the presence of a tensile hoop load.

\section{CONCLUSIONS}

For the Ares-I LH2 tank, the impact of the fail-safe design requirement results in nearly a 50\% increase in optimized tank weight for a given SBKF. The benefit of less conservative SBKF is better realized for designs that consider the fail-safe design condition, but proof test requirements may likely become the design driver for SBKF's greater than 0.75 .

\section{FUTURE WORK}

The study will be repeated for the Ares-V core stage LH2 tank. It is thought that similar trends will develop; however, the benefit of a less conservative SBKF may be greater, due to the larger ratio of compressive to tensile loading for Ares-V.

\section{REFERENCES}

1. Weingarten, V., Seide, P., Peterson, J. P., Buckling of Thin-Walled Circular Cylinders. NASA SP-8007, September 1965, revised August 1968.

2. Nemeth, M., Starnes, Jr, J., The NASA Monographs on Shell Stability Design Recommendations. NASA/TP-1998-206290, January 1998.

3. Anon.: Constellation Program Structural Design and Verification Requirements, CxP 70135, December 5, 2007.

4. Harper, D., Orthomizer User's Guide, Orthogrid Optimization Program. TBD. 


\title{
Preliminary Sizing Study of Ares-I and Ares-V Liquid Hydrogen Tanks
}

\author{
Stanley T. Oliver ${ }^{1}$ \\ NASA Marshall Space Flight Center, Huntsville, AL \\ and \\ David W. Harper ${ }^{2}$ \\ Boeing Huntsville Design Center, Huntsville, AL
}

\begin{abstract}
A preliminary sizing study of two cryogenic propellant tanks was performed using a FORTRAN optimization program to determine weight efficient orthogrid designs for the tank barrels sections only. Various tensile and compressive failure modes were considered, including general buckling of cylinders with a shell buckling knockdown factor. Eight independent combinations of three design requirements were also considered and their effects on the tanks weight. The approach was to investigate each design case with a variable shell buckling knockdown factor, determining the most weight efficient combination of orthogrid design parameters. Numerous optimization analyses were performed, and the results presented herein compare the effects of the different design requirements and shell buckling knockdown factor. Through a series of comparisons between design requirements or shell buckling knockdown factors, the relative change in overall tank barrel weights is shown. The findings indicate that the design requirements can substantually increase the tank weight while a less conservative shell buckling knockdown factor can modestly reduce the tank weight.
\end{abstract}

\section{Introduction}

FORTRAN optimization program was used to determine weight efficient orthogrid designs for the liquid hydrogen (LH2) tank barrel sections for the Ares-I and Ares-V launch vehicles. The study was performed by considering a less conservative shell buckling knockdown factor (SBKF) and permutations of specific design requirements. It is the purpose of this study to estimate the potential weight savings based on preliminary sizing methodology using a reduced SBKF. Concurrently, the investigation considers various program levied design requirements and their potential impact on weight.

\section{Background}

A metallic orthogrid panel is created by machining away material from a relatively thick plate, leaving thin integrally machined stiffeners and a thin skin. The skin forms the tank wall and the stiffeners form the orthogrid pattern. Panels are bumpformed into arc segments and several panels are welded together to form a cylindrical barrel section. A basic sketch of an orthogrid geometry is shown in Fig. 1. For this study, the stiffeners are inside the tank.

The multi-stage Ares-I and Ares-V launch vehicles are shown in Fig. 2. The Ares-I LH2 tank is near the top of the vehicle within the second stage, whereas the Ares-V LH2 tank is near the bottom of the vehicle in the first stage. These two tank configuration offer

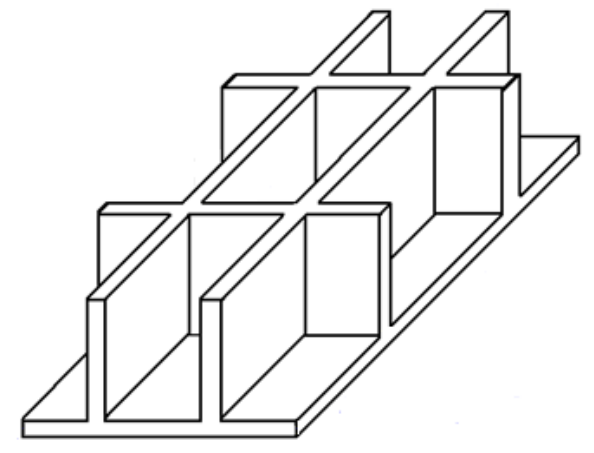

Figure 1. A generic view of an orthogrid panel geometry.

\footnotetext{
${ }^{1}$ Aerospace Engineer.

${ }^{2}$ Structural Analyst.
} 
variety for this study in terms of tank diameter, barrel lengths, and vehicle mechanical loads.

In this study, preliminary sizing refers to a design and analysis fidelity level in which the orthogrid design is smeared into an equivalent shell and ignores design features such as welds, penetrations, local reinforcements, etc. NASA SP-8007 ${ }^{1}$ provides recommended practices to determine the theoretical buckling load of circular cylinders and appropriate design specific buckling knockdown factor(s). The SBKF approach and closed-form analysis methodology found in SP-8007 represented a lower-bound of test data. This lower-bound was established with a reduced understanding of cylinder construction or testing methodologies. With significant improvements in computers and nonlinear analysis tools, more accurate buckling predictions are possible by accounting for construction sensitivities ${ }^{2}$ and testing methodology. This improved accuracy can therefore reduce the SBKF applied to the critical buckling load of circular cylinders.

\section{Design Requirements}

The design requirements were established to mitigate safety concerns and add robustness to the design. In addition to considering the flight mechanical loads and tank pressures, the following design requirements were also considered:

1) Fail-safe loss of ullage pressure (emergency condition),

2) Fixed skin thickness to carry pressure load,

3) Acceptance proof test.

The sudden loss of all ullage pressure during first stage ascent was considered a fail-safe emergency condition. Designing for the sudden loss of pressure ensured the tank would not collapse and allow for the initiation of the launch abort system. This requirement was more applicable to the Ares-I vehicle. Fail-safe emergency conditions were subject to an ultimate factor of safety of 1.0 on design loads ${ }^{3}$.

Robustness was added to the design by sizing the tank's skin to carry the pressure loads. This was to reduce the possibility that the future developed fracture properties and supporting fracture analyses would result in fracture concerns associated with the tank's skin. The fixed skin was based on the pr/t hoop stress, taking into account temperature effects on the material allowables for the flight and proof environments of -423 and 70 degrees Fahrenheit respectively.

The third requirement was the acceptance proof test. Pneumatic and mechanical loads were required to stress the tank above the design limit principal stress experienced in flight. For stability critical structures, the proof load condition is typically not a design driver; however, since less conservative SBKFs were being studied, it was possible for proof tensile stresses to become design drivers.

\section{Design Criteria}

The geometry of the integrally machined barrel panels was limited to an orthogrid configuration. The stiffeners, also designated stringers and ribs, were oriented longitudinally (stringers) and circumferentially (ribs). The overall plate thickness was limited to typical raw-plate stock for aluminum-lithium alloys. A manufacturing constraint associated with bump-forming the orthogrid panels required that the ratio of the height of a rib or stringer to its thickness be equal to or less than $10(\mathrm{~h} / \mathrm{t}<=10)$. An arbitrary constraint limited the circumferential rib spacing to 20 
inches for the Ares-I and 40 inches for Ares-V. The skin, rib and stringer thickness and height were not assigned a minimum thickness constraint.

For the compressive loading conditions, pressure stabilization was allowed using a predefined minimum pressure. Local and global buckling was not allowed at ultimate loads. For the tensile cases, maximum pressure was combined with the tensile mechanical loads and appropriate factors of safety. The same minimum and maximum pressures were used for both the Ares-I and Ares-V studies, 20 and 42 psig respectively.

The tensile and compressive failure modes considered in the optimization were:

1) Pocket or skin principal stresses (yield and ultimate)

2) Rib principal stresses (yield and ultimate)

3) Stringer principal stresses (yield and ultimate)

4) Pocket or skin buckling

5) Rib buckling

6) Stringer buckling

7) Overall cylinder buckling

The flight loads used in this study were derived from the vehicle axial and bending moment loads, combined to yield the design limit compressive and tensile mechanical line loads. The maximum and minimum pressures were considered, but the head pressure associated with the liquid hydrogen was ignored for this study due to its small contribution.

\section{Approach}

The permutations of the design requirements results in eight possible design studies:

1) Flight mechanical, fixed skin thickness, and with a proof test,

2) Flight mechanical, fixed skin thickness, and no proof test,

3) Flight mechanical, optimized skin thickness, and with a proof test,

4) Flight mechanical, optimized skin thickness, and no proof test,

5) Fail-safe loss of pressure, fixed skin thickness, and with a proof test,

6) Fail-safe loss of pressure, fixed skin thickness, and no proof test,

7) Fail-safe loss of pressure, optimized skin thickness, and with a proof test,

8) Fail-safe loss of pressure, optimized skin thickness, and no proof test.

The first four studies consider flight tension and compression design limit loads and appropriate factors of safety (1.1 for material yield and 1.4 for material ultimate). The factors of safety for pressure are 1.4 for maximum pressure and 1.0 for minimum pressure. The last four studies consider the fail-safe loss of pressure conditions, which use the same tension and compressive design limit loads used for flight, but with a factor of safety of 1.0 and no pressure stabilization. The fixed skin thickness option enforces a constant skin thickness based on hoop stress, while the optimized skin thickness option allows the skin thickness to be determined by the optimizer program, which allows the stiffeners to carry a portion of the pressure load. The proof test was an additional load case and typically only impacted the tensile failure modes.

The approach to the study used SP-8007 to establish a baseline design for each barrel and then continued reoptimizing each barrel using a progressively improved shell buckling knockdown factor. This was performed for each of the eight design studies.

\section{Optimizer Program}

A FORTRAN 77 computer program ${ }^{4}$ was utilized to obtain the minimum weight designs subjected to the constraints previously described. The orthogrid design parameters, as identified in Fig. 3, were:

1) skin thickness (t),

2) stringer spacing $\left(b_{s}\right)$,

3) rib spacing $\left(\mathrm{d}_{\mathrm{r}}\right)$,

4) stringer width $\left(t_{s}\right)$,

5) rib width $\left(\mathrm{t}_{\mathrm{r}}\right)$, and

6) stiffener height $(\mathrm{h}=\mathrm{H}-\mathrm{t})$.

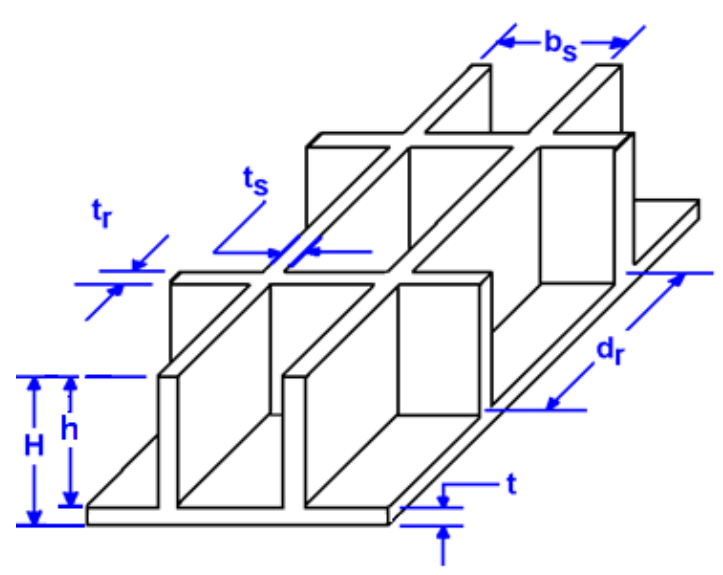

Figure 3. Orthogrid design parameters used in the optimization.

The program could process load cases comprised of 
vehicle mechanical loads, ullage pressures, head pressures, temperatures, load case specific factors of safety, and temperature-dependent material properties. It operates on the orthogid design parameters, incrementing through a user-defined vector space of parameter values, calculating margins of safety for the failure modes, and sorting through the results to find an orthogrid configuration that maintained positive margins yet had the lowest weight.

\section{Results}

A large number of optimization analyses were performed for this study. The Ares-I study had 168 barrel design optimization runs and the Ares- $\mathrm{V}$ study had 240 barrel design optimization runs, for a total of 408 barrel designs. Table 1 summarizes the number of analyses performed. The total number of studies for each tank is the product of

Table 1. Summary of Ares-I and Ares-V barrel design optimization studies.

\begin{tabular}{|c|c|c|c|}
\hline Ares-I & & Ares-V & \\
\hline Design Studies & 8 & Design Studies & 8 \\
\hline $\begin{array}{l}\text { SBKFs Considered } \\
\{\text { Baseline, } 0.65,0.70,0.75,0.80 \text {, } \\
0.85 \& 0.90\}\end{array}$ & 7 & $\begin{array}{l}\text { SBKFs Considered } \\
\{\text { Baseline, } 0.60,0.70,0.80, \& 0.90\}\end{array}$ & 5 \\
\hline Barrel Sections & 3 & Barrel Sections & 6 \\
\hline Ares-I Total & 168 & Ares-V Total & 240 \\
\hline & & Total & 408 \\
\hline
\end{tabular}

the number of Design Studies, SBKFs Considered and Barrel Sections. The 0.65, 0.75 and 0.85 SBKFs were not considered for the Ares- $\mathrm{V}$ study to reduce the number of optimization runs.

Figure 4 shows a point cloud plot of the design results from this study. Note, the Ares-I results for SBKFs of $0.65,0.75$ and 0.85 are not shown. Also shown is a curve that represents the lower bound axial knockdown factor from SP-8007. Each data point represents the SBKF versus the R/t $\mathrm{t}_{\mathrm{eff}}$ for each Ares-I and Ares-V tank barrel design, where

$$
\mathrm{t}_{\text {eff }}=\sqrt[4]{\frac{144 \cdot \mathrm{D}_{11} \cdot \mathrm{D}_{22}}{\mathrm{~A}_{11} \cdot \mathrm{A}_{22}}}
$$

\section{Point Cloud of Ares-I and Ares-V LH2 Tank Barrel Optimization Results}

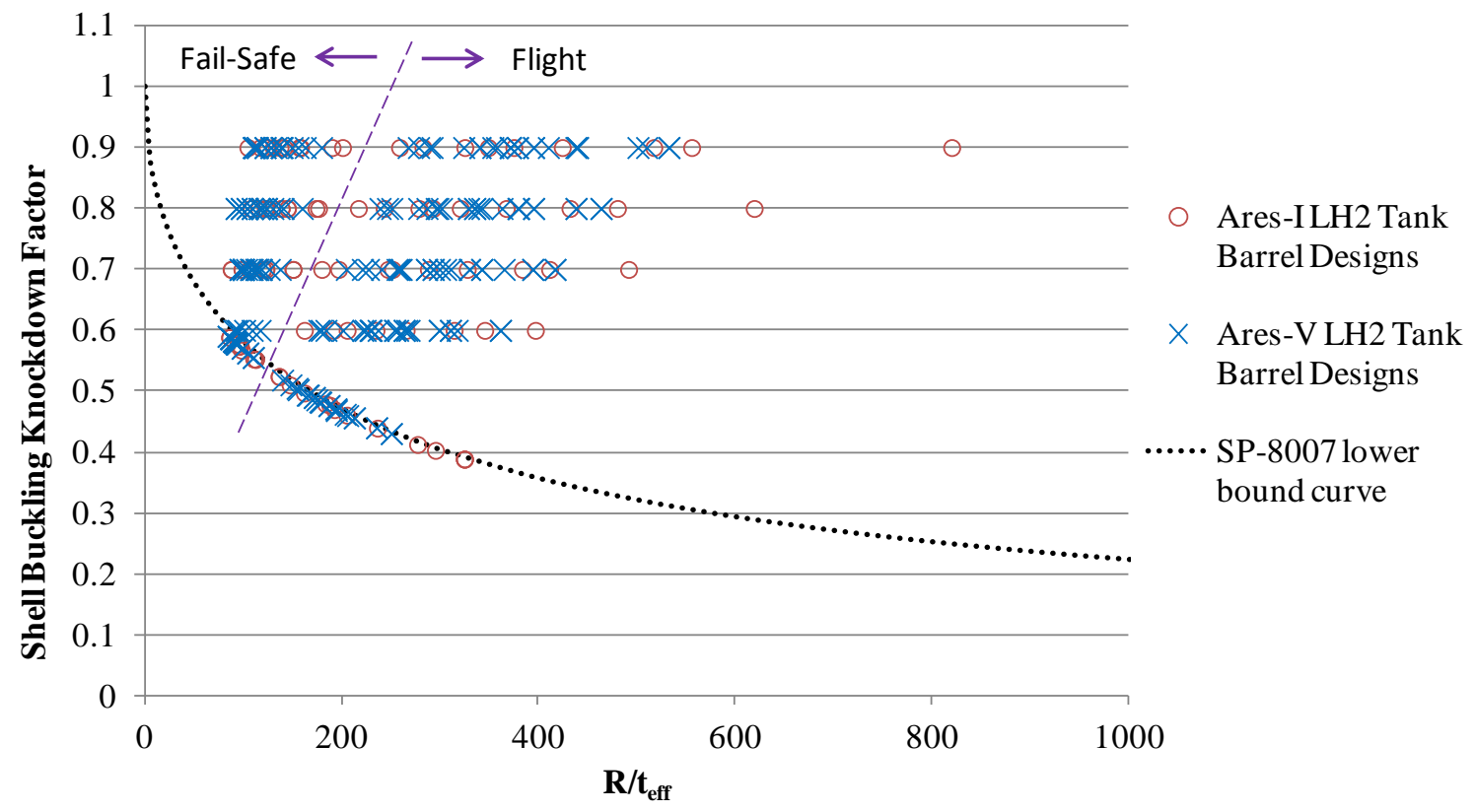

Figure 4. Point cloud of optimized barrel designs with $\mathrm{SBKF}$ as a function of $R / t_{\text {eff }}$ 
and $\mathrm{R}$ is the radius of the cylinder. The $\mathrm{A}_{\mathrm{ii}}$ and $\mathrm{D}_{\mathrm{ii}}$ stiffness coefficients, which are based on the orthogrid geometry, were found using equations from Reference 1. The Ares-I and Ares-V results are dispersed, with no clear distinction between the two tanks. Therefore, the effects corresponding to differences between the barrel lengths, magnitude of loads and/or tank radius do not appear to affect the results. However, there does appear to be a division in the groupings for the fail-safe results and flight results, shown as a dotted line through the point cloud in Fig. 4. The Ares-I and Ares-V data points on the SP-8007 curve represent the baseline design options for the three Ares-I barrels and six Ares-V barrels for each of the eight design studies. The remaining data points represent the reoptimized designs using the corresponding SBKFs. The loads do not change, only the knockdown factor associated with general overall cylinder buckling. The trend in the optimization study is lighter weight designs result from less conservative SBKFs. The reduction in weight is primarily due to a decrease in stiffener height and thickness.

It should be pointed out that the few Ares-I design points on the right side of Figure 4 that appear to be slightly out of the main grouping represent the forward barrel for design case 1, (flight mechanical, fixed skin thickness, and with a proof test), which had the lowest compression loading. Recall in the Design Criteria section that a minimum thickness criterion was not established for skin and stiffener height and thickness. Therefore, this allowed the optimization result to consider relatively thin stiffeners, which trended these results to the right.

The next sets of results are based on the summation of barrel section weights for the Ares-I or Ares-V LH2 tank. However, no weld lands, rings, domes, etc. were considered. The tank weights were normalized with respect the baseline heaviest tank, the fail-safe with fixed skin thickness design that utilized the SP-8007 knockdown factors.

\section{A. Ares-I}

The normalized Ares-I LH2 tank weight as a function of shell buckling knockdown factor is shown in Figure 5. It should be noted that there are six results curves shown in Figure 5, but there were eight possible design studies. For the cases with a fixed skin thickness, the proof test had no impact on the weights. Therefore, those cases yielded the same results, and the legend in the graph indentifies the two corresponding cases as combined for both the failsafe and flight design cases. In addition, the baseline SBKF values for Ares-I barrels were typically lower than 0.65, but 0.65 represents the lowest common SBKF for the three barrel sections for all Ares-I studies.

There are two distinct groupings in Figure 5, the closely spaced fail-safe grouping and the more widely spaced flight grouping. The fail-safe grouping's weight is higher because the optimization could not utilize pressure stabilization; whereas for flight, minimum pressure stabilization was sufficient to provide substantial benefit, even with the higher factors of safety applied to the flight loads.

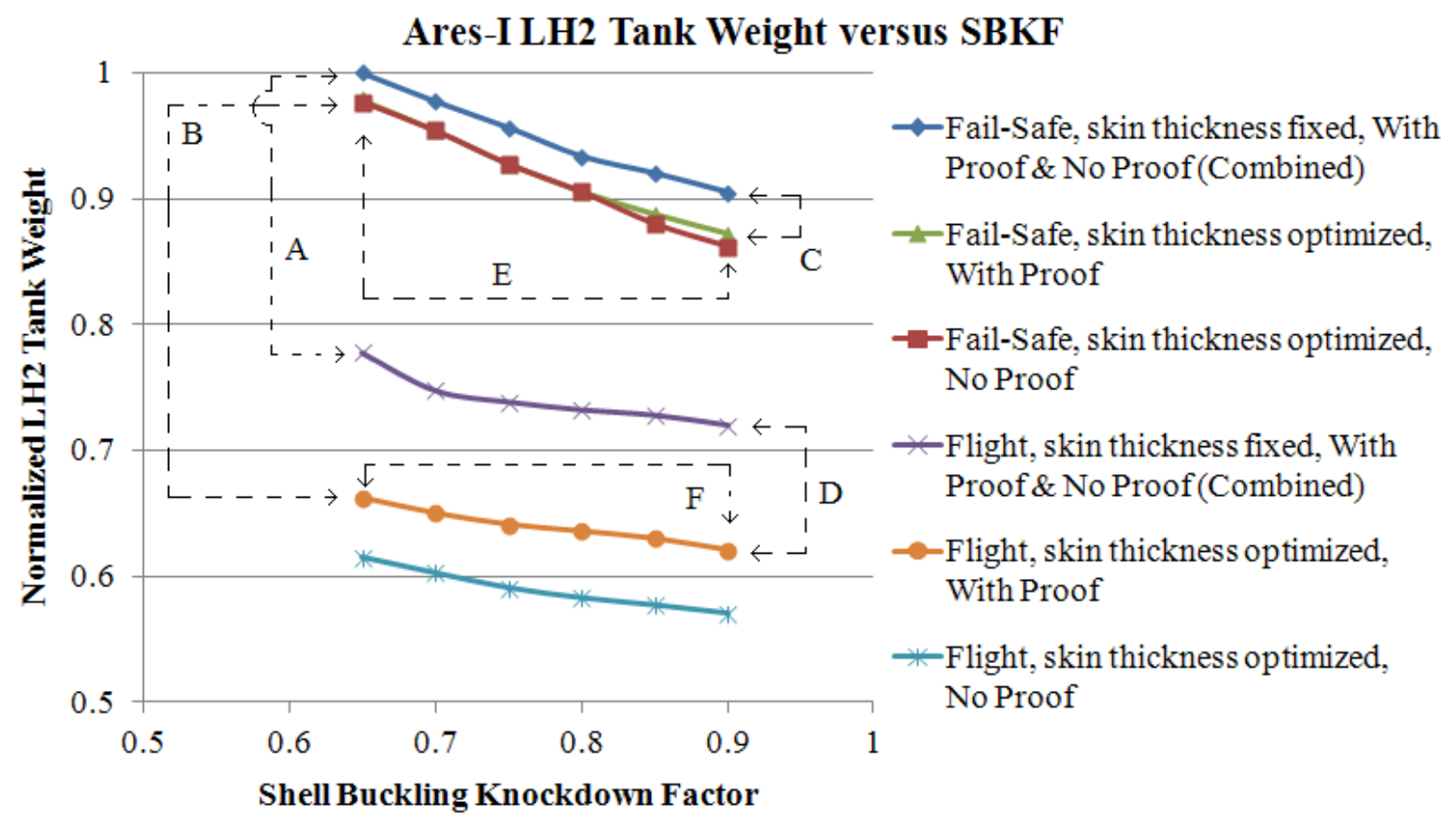

Figure 5. Optimization results for Ares-I LH2 tank for various design considerations. 
Surveying the Ares-I LH2 tank normalized results for a given shell buckling knockdown factor, 0.65 for example, shows that the fail-safe requirement for the fixed skin thickness designs could result in a $29 \%$ increase in weight over the flight design for the fixed skin thickness (comparison A). The same comparison can be made for maintaining a proof test, yet allowing the skin thickness to be optimized. Implementing the fail-safe design requirement resulted in a weight increase of $47 \%$ over the corresponding flight design (comparison B).

Enforcing a fixed skin thickness design results in additional weight compared to allowing the skin thickness to be determined through optimization. It is unlikely that a proof test would not be required for launch vehicles; therefore, the following two comparisons assume a proof test and compare the weight impact of skin thickness for the fail-safe and flight cases. Assuming a shell buckling knockdown factor of 0.90 , the fail-safe design with fixed skin thickness increased the weight by $4 \%$ over an optimized skin design (comparison C). For the flight cases, a $16 \%$ increase in weight from an optimized skin to a fixed skin (comparison D). It should be noted that for the failsafe cases with optimization for the skin thickness, the proof test began to be a design driver for SBKFs greater than 0.80 .

Another comparison looked at the effect of utilizing a less conservative SBKF. For a fail-safe case, allowing the skin thickness to vary and accounting for a proof test, the potential weight savings of designing for a SBKF of 0.90 compared to 0.65 was $12 \%$ (comparison E). For a flight case, allowing the skin thickness to vary and accounting for a proof test, the potential weight savings of designing for a SBKF of 0.90 compared to 0.65 was $7 \%$ (comparison F). One can see from the graph that the effect of SBKF is less for the flight cases than the fail-safe cases (observed slope of data).

\section{B. Ares-V}

The normalized Ares-V LH2 tank weight as a function of shell buckling knockdown factor is shown in Figure 6. Similar to Ares-I results, the fail-safe, fixed skin thickness, proof and no proof cases were combined as well as the two similar cases for flight. Unlike the Ares-I, the Ares-V fail-safe, skin thickness optimized cases, proof and no proof cases did not appear to develop a separation at the higher SBKFs. The baseline SBKF values for barrel section were typically lower than 0.60 , but 0.60 represents the lowest common SBKF for all six barrel sections for all Ares-V studies.

Surveying the Ares-V LH2 tank normalized results for a given shell buckling knockdown factor, 0.60 for example, shows that the fail-safe requirement for the fixed skin thickness designs could result in a $39 \%$ increase in

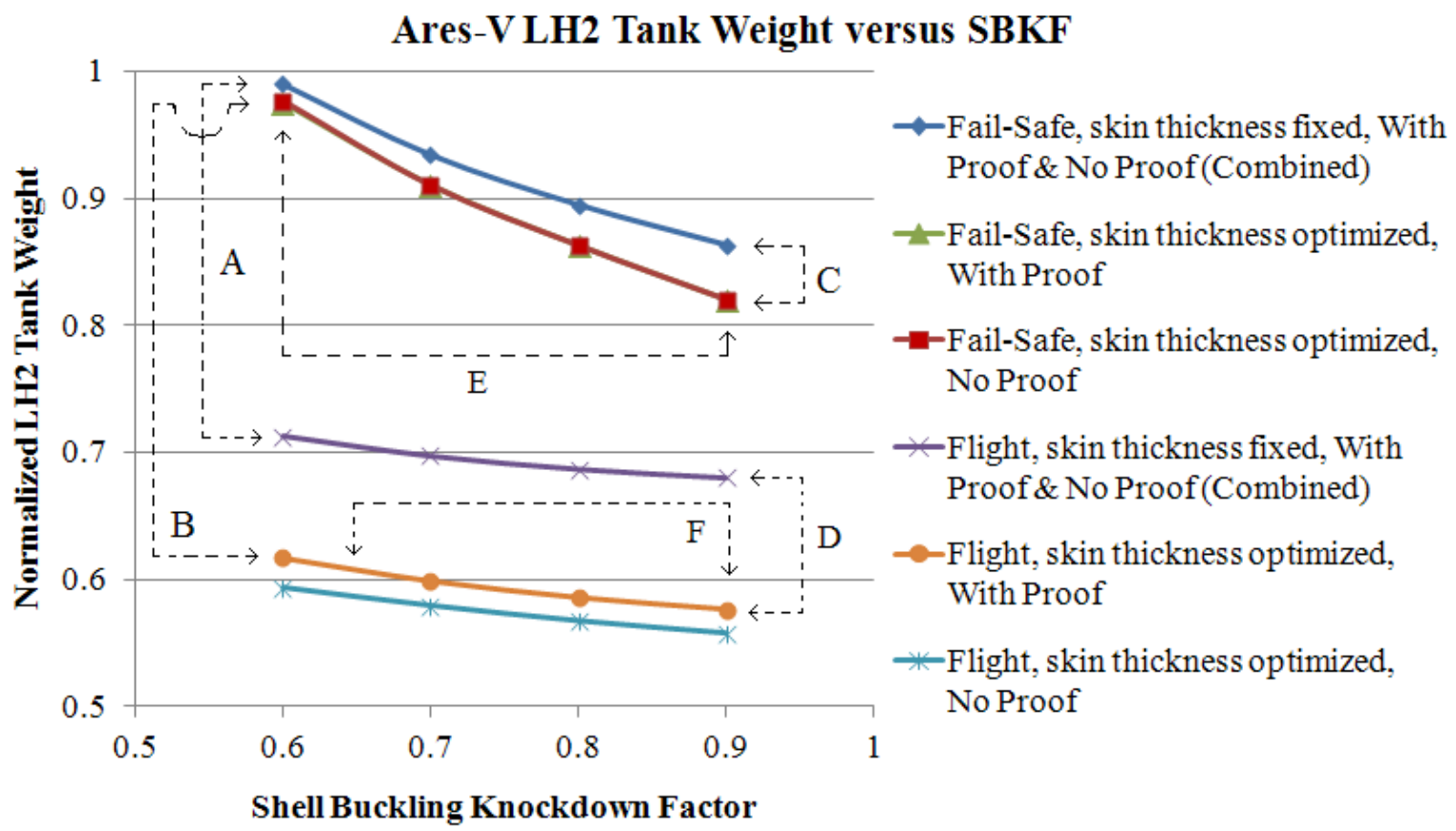

Figure 6. Optimization results for Ares-V LH2 tank for various design considerations. 
weight over the flight design for the fixed skin thickness (comparison A). The same comparison can be made for maintaining a proof test, yet allowing the skin thickness to be optimized. Implementing the fail-safe design requirement resulted in a weight increase of $58 \%$ over the corresponding flight design (comparison B).

Enforcing a fixed skin thickness design results in additional weight compared to allowing the skin thickness to be determined through optimization. It is unlikely that a proof test would not be required for launch vehicles; therefore, the following two comparisons assume a proof test and compare the weight impact of skin thickness for the fail-safe and flight cases. Assuming a shell buckling knockdown factor of 0.90 , the fail-safe design with fixed skin thickness increased the weight by 5\% over an optimized skin design (comparison C). For the flight cases, an $18 \%$ increase in weight from an optimized skin to a fixed skin (comparison D).

Another comparison looked at the effect of utilizing a less conservative SBKF. For a fail-safe case, allowing the skin thickness to vary and accounting for a proof test, the potential weight savings of designing for a SBKF of 0.90 compared to 0.60 was $19 \%$ (comparison E). For a flight case, allowing the skin thickness to vary and accounting for a proof test, the potential weight savings of designing for a SBKF of 0.90 compared to 0.60 was $7 \%$ (comparison F). One can see from the graph that the effect of SBKF is less for the flight cases than the fail-safe cases (observed slope of data).

\section{Conclusions}

A preliminary sizing study of two cryogenic propellant tanks was performed using a FORTRAN optimization program to determine weight efficient orthogrid designs for the tank barrels sections. Various tensile and compressive failure modes were considered, including general buckling of cylinders with a shell buckling knockdown factor. Eight independent combinations of three design requirements were also considered and their effects on the tanks weight. Numerous optimization analyses were performed, and the preliminary sizing findings indicate that a fail-safe requirement has a tremendous effect on the barrel weights of both the Ares-I and Ares-V tanks. Enforcing a fixed skin thickness as well as a proof test resulted in a more modest increase in tank weight, some of which could have been the as a result of not establishing a minimum thickness requirement for the skin and stiffeners. The effect of a less conservative knockdown factor resulted in a savings of less than $10 \%$, but worth considering for design purposes.

\section{Future Work}

A potential follow-up effort would concentrate on case 3 (flight, optimized skin thickness, and account for proof test), a likely design scenario for future launch vehicles. The study would investigate the effect on barrel weight while using lower factors of safety for pressure (1.25 instead of 1.4). In addition, the study could include an investigation on the tank weight while allowing local buckling at limit load for skin and stiffeners. Both studies would include lower bound thickness criteria for skin and stiffeners.

\section{References}

${ }^{1}$ Weingarten, V., Seide, P., Peterson, J. P., Buckling of Thin-Walled Circular Cylinders. NASA SP-8007, September 1965, revised August 1968.

${ }^{2}$ Nemeth, M., Starnes, Jr, J., The NASA Monographs on Shell Stability Design Recommendations. NASA/TP-1998-206290, January 1998.

${ }^{3}$ Anon.: Constellation Program Structural Design and Verification Requirements, CxP 70135, December 5, 2007.

${ }^{4}$ Harper, D., “Orthomizer User's Guide, Orthogrid Optimization Program”, Marshall Space Flight Center, EV31, 2010 (unpublished). 